\title{
Molecular characterization and phylogenetic analysis of fowl adenovirus serotype-4 from Guangdong Province, China
}

Fu Yuming ${ }^{1}$, Yuan Sheng ${ }^{1}$, Deng Wenyu ${ }^{2}$, Chi Shihong ${ }^{1}$, Li Wenfeng ${ }^{1}$, Huang Wenjing ${ }^{1}$, Li Xiaowen ${ }^{1}$, Saeed El-Ashram ${ }^{1,3}$ (iD, Kun Mei ${ }^{1}$, Guo Jinyue ${ }^{1}$, Zhang Xuelian ${ }^{1}$, Li Zhili and Huang Shujian ${ }^{1}$

1. Department of Microbiology, College of Life Science and Engineering, Foshan University, Foshan 528231, Guangdong Province, China; 2. Guangdong Women and Children Hospital, Guangdong Province, China; 3. Department of Zoology, Faculty of Science, Kafrelsheikh University, Kafr el-Sheikh 33516, Egypt.

Corresponding author: Huang Shujian, e-mail: huangshujian@fosu.edu.cn

Co-authors: FY: fym3551256@gmail.com, YS: fsyuans@126.com, DW: 48684355@qq.com,

CS: 1041738317@qq.com, LW: liwenfengfky@163.com, HW: 757586187@qq.com, LX: 18306616234@163.com, SE: saeed_elashram@yahoo.com, KM: meikun0000@live.com, GJ: guojinyue12@163.com, ZX: zxIsjw0312@163.com, LZ: pinganzhili@163.com

Received: 26-11-2019, Accepted: 09-04-2020, Published online: 24-05-2020

doi: www.doi.org/10.14202/vetworld.2020.981-986 How to cite this article: Yuming $F$, Sheng $Y$, Wenyu $D$, Shihong $C$, Wenfeng L, Wenjing H, Xiaowen L, El-Ashram S, Mei K, Jinyue G, Xuelian Z, Zhili L, Shujian H (2020) Molecular characterization and phylogenetic analysis of fowl adenovirus serotype-4 from Guangdong Province, China, Veterinary World, 13(5): 981-986.

\begin{abstract}
Aim: Our aim in this study was to isolate potentially novel strains of fowl adenovirus serotype-4 (FAdV-4) that is currently circulating in broiler chicken flocks in Guangdong Province, China, and to compare nucleotide and amino acid (AA) sequences of their respective hexon genes.
\end{abstract}

Materials and Methods: The experiment was carried out on poultry farms experiencing outbreaks of FAdV-4-associated hydropericardium syndrome (HPS). Tissue samples from the hearts and livers of deceased chickens were screened for FAdV-4 infection using hexon gene-specific polymerase chain reaction (PCR).

Results: New virus isolates were used to infect 7-day-old chicks, which went onto reproduce typical HPS signs. The hypervariable region of the FAdV-4 hexon gene was PCR-amplified and sequenced. The hexon nucleotide and deduced AA sequence identities were $99.8-99.9 \%$ and $99.5-99.8 \%$, respectively, among the four novel isolates. In addition, the new isolates were $97-100 \%$ and $96.4-99.9 \%$ identical to the nucleotide and deduced AA sequences, respectively, of FAdV-4 hexon genes available in the National Center for Biotechnology Information GenBank database. Phylogenetic analyses, based on the hexon gene sequence, revealed that the new isolates, clustered with FAdV-C; the FAdV-A, FAdV-B, FAdV-D, and FAdV-E viruses, were more distantly related.

Conclusion: New FAdV-4 isolates from Guangdong Province are similar to those identified in other regions of the world. This information provides critical insight into HPS epidemiology and provides a perspective for monitoring outbreaks and developing strategies for disease prevention.

Keywords: Angara disease, fowl adenovirus-4, phylogenetic analysis.

\section{Introduction}

Adenoviruses comprise a large group of DNA viruses that infect humans, animals, and birds [1]. Fowl adenoviruses (FAdVs) are members of the genus Aviadenovirus of the family Adenoviridae. Aviadenoviruses are associated with a variety of diseases, including inclusion body hepatitis (IBH), hydropericardium syndrome (HPS), gizzard erosions, proventriculitis, and tenosynovitis [2]. Aviadenoviruses have been subdivided into five species with 12 serotypes based on their molecular structures and cross-neutralization test results, respectively [3]. There are currently five known FAdV species, including FAdV A (FAdV

\footnotetext{
Copyright: Yuming, et al. Open Access. This article is distributed under the terms of the Creative Commons Attribution 4.0 International License (http://creativecommons.org/licenses/ by/4.0/), which permits unrestricted use, distribution, and reproduction in any medium, provided you give appropriate credit to the original author(s) and the source, provide a link to the Creative Commons license, and indicate if changes were made. The Creative Commons Public Domain Dedication waiver (http:// creativecommons.org/publicdomain/zero/1.0/) applies to the data made available in this article, unless otherwise stated.
}

serotype 1), FAdV B (FAdV serotype 5), FAdV C (FAdV serotypes 4 and 10), FAdV D (FAdV serotypes 2, 3, 9, and 11), and FAdV E (FAdV serotypes 6, 7, 8a, and $8 \mathrm{~b}$ ) [4]. IBH and HPS have been reported by poultry farms from various parts of the world, including within China. FAdV serotype-4 (FAdV-4) has been identified as the causative agent of HPS [5], also known as Angara disease [6].

Clinical HPS cases have been reported since 2015 in poultry farms in China, including those in Shandong, Hubei, Jiangsu, Anhui, Jiangxi, and Henan Provinces; this represents an enormous economic loss for the domestic poultry industry [7]. There has been little to no molecular characterization or phylogenetic analyses of FAdV strains currently circulating in broiler chicken flocks located in Guangdong Province.

The aim of the study was to isolate FAdV-4 strains from chickens in Guangdong Province that were clinically diagnosed with HPS and to investigate the similarities and differences among the nucleotide 
sequences of their respective hexon genes. A more complete understanding of specific FAdV-4 sequences will provide epidemiological information that may lead to improve disease prevention strategies through effective vaccination.

\section{Materials and Methods}

\section{Ethical approval}

All experiments were conducted according to the ethical standards and protocols approved by the Committee of Animal Experimentation of College of Life Science and Engineering, Foshan University, Guangdong, China (permission number 2016- FOSU-CLSE21).

\section{Collection of samples}

Four tissue samples, including hearts and livers of dead chickens with suspected HPS (Angara disease), were collected from five poultry farms in Guangdong Province, China. Signs of disease included pericardial effusion, epicardial fat deposits, and bleeding. The livers were enlarged and fragile with discrete petechial hemorrhages. The samples were stored at $-20^{\circ} \mathrm{C}$ until use.

\section{Virus isolation}

The tissue samples $(0.2 \mathrm{mg})$ were pulverized; the resulting material was suspended in saline and used to inoculate 9-day-old embryonated chicken eggs (ECEs). Normal saline, without liver tissue, was used as a negative control. The ECEs were examined every $12 \mathrm{~h}$ after inoculation; the dead embryos were harvested and examined for pathological changes. Allantoic fluids were collected for repeated inoculation of chicken embryos in order to amplify virus [7].

\section{Hemagglutination (HA) and dipstick tests}

To examine virus-mediated HA reactions, allantoic fluids from chick embryos from eggs that were inoculated with liver tissue were added to chicken, rat, guinea pig, and sheep red blood cells [8]. A nucleic acid dipstick test was used to detect avian influenza (AI) and avian leukosis virus (ALV) [9].

\section{Genomic DNA extraction and hexon gene amplification}

Allantoic fluid samples were subjected to centrifugation at $3000 \mathrm{rpm}$ for $10 \mathrm{~min}$; viral DNA was isolated directly from this material using a Viral RNA/DNA Purification kit (Thermo Fisher Scientific China Co Ltd.), according to the manufacturer's instructions and details noted in El-Ashram et al. [10]. A 1008-bp fragment of the hexon gene (GenBank No. EU931692.1) was amplified by polymerase chain reaction (PCR) to identify samples. The primers designed for this purpose are listed in Table-1. The PCR reaction included $2 \times$ Easy Taq PCR SuperMix, 10 pmol of each primer, and $2 \mu \mathrm{L}$ of DNA template and nuclease free water to $25 \mu \mathrm{L}$. The following PCR conditions were used: Initial denaturation at $94^{\circ} \mathrm{C}$ for $4 \mathrm{~min}, 30$ cycles of denaturation at $94^{\circ} \mathrm{C}$ for $30 \mathrm{~s}$, annealing at $66^{\circ} \mathrm{C}$ (for fragment A) or $57^{\circ} \mathrm{C}$ (for fragment $\mathrm{B}$ ) for $30 \mathrm{~s}$, extension at $72^{\circ} \mathrm{C}$ for $60 \mathrm{~s}$, and a final extension at $72^{\circ} \mathrm{C}$ for $10 \mathrm{~min}$.

\section{DNA cloning and sequencing}

PCR amplicons were subjected to agarose gel electrophoresis, excised from the gel, purified using an Agarose Gel DNA Purification Kit (TaKaRa, Japan), and cloned into the pMD18-T vector according to the manufacturer's instructions (TaKaRa, Japan). The recombinant plasmid was transformed into DH5 $\alpha$ competent E. coli cells. Plasmid DNA was purified using the E.Z.N.A. ${ }^{\circledR}$ Plasmid Mini Kit I (Omega Bio-Tek, USA) and quantified by spectrophotometric analysis. Recombinant plasmids of fragment A or fragment B were sequenced by Shanghai Sangon Bioengineering Ltd., China with primers included in Table-1. Detailed information, including the GenBank accession numbers of all sequences of the four novel strains, is available in Table-2. ClustalX software (version 1.83, Desmond G. Higgins, Paul Sharp, Trinity College Dublin, Ireland) was used to construct multiple alignments. Phylogenetic trees were constructed using MEGAsoftware version 5.1 (https://mega.software.informer.com/5.1/), by the neighbor-joining analysis using the Maximum Composite Likelihood algorithm with 1000 bootstrap replicates. The datasets used for phylogenetic datasets included four novel FAdV-4 isolates from this study and 25 reference strains retrieved from GenBank database (Table-3).

\section{Macroscopic examination and molecular identifica- tion of FAdV-4 isolates}

The pathogenicity of the newly isolated FAdV-4 strains was evaluated in 7-day-old specific-pathogen-free (SPF) chickens through intramuscular inoculation of $0.2 \mathrm{~mL}$ allantoic fluid from each infected, 9-day-old ECE. Negative controls included 7-day-old SPF chickens that were inoculated intramuscularly with phosphate-buffered saline. Heart and liver samples were collected from deceased birds for further evaluation and virus identification.

\section{Results}

Macroscopic examination of naturally-infected broiler chickens

Broiler chickens infected with FAdV-4 showed signs of morbidity, including loss of appetite and lethargy, associated with loose, yellow-green-colored stools. The most common gross lesion at necropsy was gelatinous material accumulating in the pericardial cavity. Other findings included swollen livers with petechial hemorrhages. No gross lesions were detected in uninfected chickens (Figure-1).

Table-1: Sequences of primers employed in the conventional PCR.

\begin{tabular}{llll}
\hline Target genes & $\begin{array}{l}\text { Primer expected } \\
\text { product }\end{array}$ & bp & Temp \\
\hline Hexon gene of FAdV-4 & F: CCTCCAACAG & 1008 & $47^{\circ} \mathrm{C}$ \\
& $\begin{array}{l}\text { TTCATTT } \\
\text { R: TCTTCGTAAC } \\
\text { CGTCATT }\end{array}$ & & \\
& & & \\
\hline
\end{tabular}

FAdV-4=Fowl adenovirus species C serotype-4, $\mathrm{PCR}=$ Polymerase chain reaction 
Table-2: GenBank accession numbers for all sequences of four strains.

\begin{tabular}{lllllc}
\hline S. No. & Date & Host & Age & Strain name & $\begin{array}{c}\text { Deposited in GenBank with } \\
\text { accession numbers }\end{array}$ \\
\hline 1 & May 17, 2016 & Chicken & $\sim 20-30$ days & CH/GDBL-S1/2016 & MK875246 \\
2 & February 25, 2017 & Chicken & 3 days & CH/GDHZ-S2/2017 & MK875247 \\
3 & February 14, 2017 & Chicken & 100 days & CH/GDJM-S6/2017 & MK875248 \\
4 & November 14, 2017 & Chicken & 60 days & CH/GDHZ-S7/2017 & MK875249 \\
\hline
\end{tabular}

Table-3: Details of fowl aviadenovirus strains and isolates employed for sequence alignment and phylogenetic analysis.

\begin{tabular}{llllc}
\hline S. No. & Collection date & Name of strains & Geographic origin & GenBank accession no. hexon gene \\
\hline 1 & January-1996 & VR-432 & Phelps & NC_001720.1 \\
2 & November-2000 & JM1/1 & Japan: Kagoshima & MF168407.1 \\
3 & November-1995 & CELO & Russia & Z67970.1 \\
4 & July-2003 & Fowl aviadenovirus A & UK & AC_000014.1 \\
5 & Early 1970s & 340 & Ireland & NC_021221.1 \\
6 & Early 1970s & 340 & Ireland & KC493646.1 \\
7 & September-2015 & C-2B & USA & KT717889.1 \\
8 & July-2004 & ON1 & Canada & NC15323.1 \\
9 & September-2015 & ZK & China & KU647689.1 \\
10 & October-2015 & HN/151029 & China & KX090424.1 \\
11 & September-2015 & SDXT2-15 & China & KU877432.1 \\
12 & February-2015 & HB1502 & China & KX421401.2 \\
13 & May-2002 & Fowl aviadenovirus 4 & Indian & AJ459805.1 \\
14 & March-2010 & Kr-Gunwi & South Korea & KP295409227.1 \\
15 & 1995 & MX-SHP95 & Mexico & KJ207054.1 \\
16 & October-2009 & Fowl aviadenovirus C & Russia & KU175344.1 \\
17 & January-2014 & FAdV/PA/Layer/27614/13 & USA & NC_000899.1 \\
18 & August-1998 & A-2A & Canada & KU497449.1 \\
19 & July-2015 & LN/1507 & China & KU746335.1 \\
20 & January-1995 & MX95-S11 & Mexico & AC_000013.1 \\
21 & July-2003 & Fowl aviadenovirus D & UK & KT862809.1 \\
22 & $1950 s / 1960 s$ & YR36 & Japan & JN112373.1 \\
23 & December-2009 & 764 & Canada & KX077988.1 \\
24 & November-2015 & HLJ/151129 & China & KU517714.1 \\
25 & 2004 & UPM04217 & Malaysia &
\end{tabular}

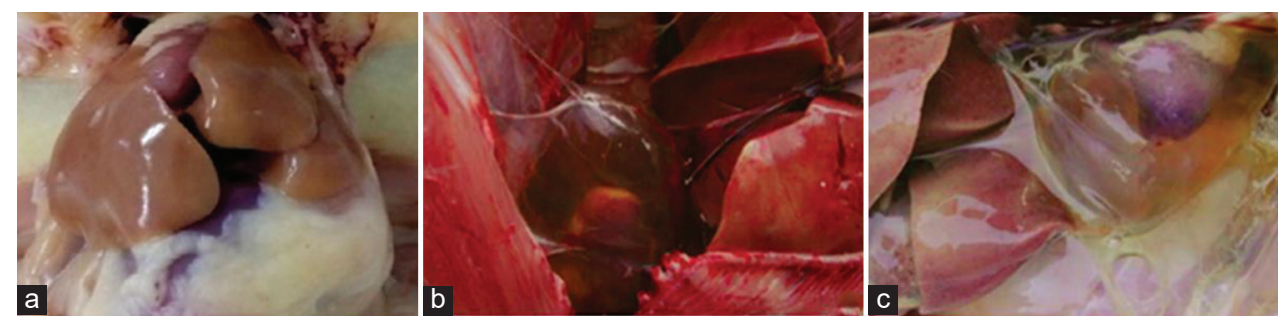

Figure-1: Prominent pathological changes in the fowl adenovirus species $C$ serotype-4-infected chickens (b and $c$ ) compared to the uninfected controls (a).

Infection of colon epithelial cells (CECs) with FAdV-4

CECs were infected with pulverized liver materials; allantoic fluid was collected; and virus infection was evaluated. No red blood cell aggregation was detected through a HA test. A nucleic acid test strip assay was negative for AI and ALV. PCR amplification with FAdV-4 hexon gene-specific primers generated a band of approximately 1008 bp (Figure-2).

Macroscopic examination of experimentally-infected chicks and molecular identification of FAdV-4 isolates

The gross changes, identified on necropsy of the experimentally-infected chicks, were consistent with those observed in response to natural infection. The lesions were typical for HPS and included gelatinous material accumulations in the pericardial cavity (Figure-3). No deaths were recorded among the chickens in the uninfected control group. We used the primers listed in Table-2 to amplify a fragment of the hexon gene of FAdV-4 virus; liver and heart tissue samples were FAdV-positive by PCR; no amplification was detected in tissues from the uninfected controls.

\section{Sequence alignment and phylogenetic analysis}

We identified four novel isolates of FAdV-4 from infected broiler chickens. The hexon genes of each strain include 2814 nucleotides that encode 937 amino acids (AAs). The highly variable regions, based on those identified in the reference SDXT2 (GenBank KU877432.1) strain, included nucleotides 
90 200, 988 1233, 2017 2067, and 2650 2679, encoding AAs 330 411, 663 667, 673 689, and 887 893. Among the regions of sequence divergence, we note nine specific AA changes, including A196R, A197G, Y989N, N1042D, D1231N, T2063I, L2066P, M2659V, and L2678P (Table-4).

The nucleotide sequences of the amplified hexon genes and their deduced AA sequences were 99.8-99.9\% and $99.5-99.8 \%$ identical to one another, respectively. Similarly, the nucleotide and deduced AA sequences revealed 97-100\% (96.4-99.9\%) identity with FAdV-4 gene sequences (FAdV-C strains)

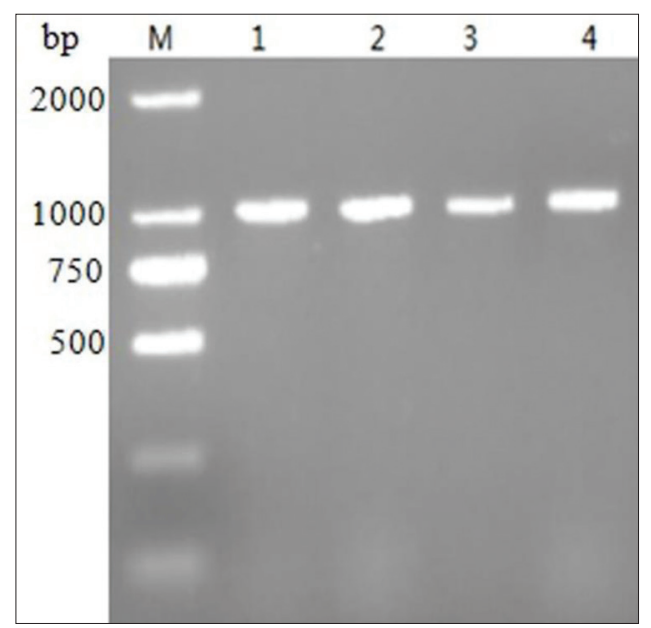

Figure-2: Amplification result of the partial hexon gene. M, 2000 bp DNA Marker, lanes (CH/GDBL-S1/2016, CH/ GDHZ-S2/2017，CH/GDJM-S6/2017，CH/GDHZ-S7/2017， respectively).

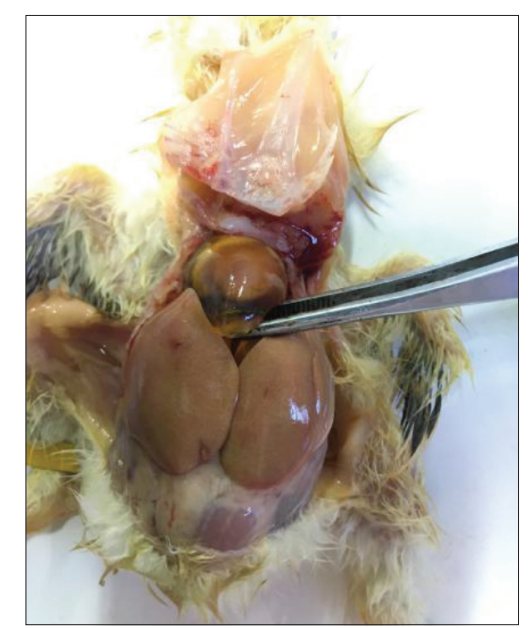

Figure-3: Gross pathology of fowl adenovirus species C serotype-4-infected chicks at necropsy.

Table-4: Amino acid point mutations of the Chinese strains compared to SDXT2 (KU877432.1).

\begin{tabular}{lllllllll}
\hline \multirow{2}{*}{ Strains } & \multicolumn{6}{c}{ Positions of amino acid point mutations } \\
\cline { 2 - 8 } & $\mathbf{6 6}$ & $\mathbf{3 3 0}$ & $\mathbf{3 4 8}$ & $\mathbf{4 1 1}$ & $\mathbf{6 8 8}$ & $\mathbf{6 8 9}$ & $\mathbf{8 8 7}$ & $\mathbf{8 9 3}$ \\
\hline SDXT2 & $\mathrm{A}$ & $\mathrm{Y}$ & $\mathrm{N}$ & $\mathrm{D}$ & $\mathrm{T}$ & $\mathrm{L}$ & $\mathrm{M}$ & $\mathrm{L}$ \\
$\mathrm{S} 1$ & $\mathrm{~A} \rightarrow \mathrm{R}$ & - & - & $\mathrm{D} \rightarrow \mathrm{N}$ & $\mathrm{T} \rightarrow \mathrm{I}$ & - & - & - \\
$\mathrm{S} 2$ & $\mathrm{~A} \rightarrow \mathrm{G}$ & - & $\mathrm{N} \rightarrow \mathrm{D}$ & $\mathrm{D} \rightarrow \mathrm{N}$ & $\mathrm{T} \rightarrow \mathrm{I}$ & $\mathrm{L} \rightarrow \mathrm{P}$ & $\mathrm{M} \rightarrow \mathrm{V}$ & - \\
$\mathrm{S} 3$ & - & $\mathrm{Y} \rightarrow \mathrm{N}$ & - & $\mathrm{D} \rightarrow \mathrm{N}$ & $\mathrm{T} \rightarrow \mathrm{I}$ & - & - & - \\
S4 & $\mathrm{A} \rightarrow \mathrm{G}$ & - & - & $\mathrm{D} \rightarrow \mathrm{N}$ & $\mathrm{T} \rightarrow \mathrm{I}$ & - & - & $\mathrm{L} \rightarrow \mathrm{P}$ \\
\hline
\end{tabular}

retrieved from the National Center for Biotechnology Information GenBank database; by contrast, their nucleotide and AA sequences were $76.0-76.3 \%$ (80.4-81\%), 73.2-73.3\% (76.8-77.1\%), 69.6-71.7\% (64.2-77.7\%), and 74.8-75.1\% (77.6-78.2\%) identical to those of the FAdV-A, FAdV-B, FAdV-D, and FAdV-E strains, respectively. Phylogenetic analysis, based on the hexon gene sequence, revealed that the new isolates were most closely related to the reference FAdV-C strains; FAdV-A, FAdV-B, FAdV-D, and FAdV-E strains clustered in different branches of the phylogenetic tree (Figure-4).

\section{Discussion}

HPS was first described in 1987 in broiler chickens in Karachi, Pakistan. Typical macroscopic HPS lesions include a gelatinous substance accumulating in the pericardial sac, accompanied by an enlarged liver [11]; the viscera of infected animals undergo varying degrees of bleeding [12]. The findings in our current study are consistent with these disease descriptions $[13,14]$. FAdVs are identified routinely in chickens on poultry farms throughout the world. Before 2014, circulating strains of FAdV-4 were recorded in China, although no outbreaks of severe HPS were reported [15]. Since July 2015, HPS outbreaks that are associated with high rates of morbidity and mortality [14] have occurred. HPS has been reported throughout world, including in India, Canada, Hungary, Korea, Japan, and Poland [7]. The emergence of hepatitis-HPS in China has been attributed to FAdV species C serotype-4 (FAdV-4) [16]; FAdVs remain in circulation among broilers, domestic chickens, and egg-layers, resulting in serious economic losses in the poultry industry [16].

In this study, four novel FAdV-4 strains were isolated from tissues from diseased and deceased chickens suspected of having HPS (also known as Angara disease). We subjected these isolates to experimental validation and conventional PCR assays. Among our findings, the gross pathology was consistent with that observed in earlier reports. All four of the pathogenic FAdV strains isolated in this study were genetically related to serotype- 4 based on the sequence analysis of the amplified hexon gene; this is currently the dominant serotype circulating in Guangdong Province, China [16]. Our results suggest that continued surveillance of FAdVs is needed to monitor the spread of this virus and virus-associated HPS.

The novel FAdV-4 isolates identified in this study are highly homologous to one another, with $99.8-99.9 \%$ nucleotide sequence homology within the amplified hexon gene. The FAdV-4 isolates were also closely related to both domestic and foreign FAdV-C strains, displaying the $97-100 \%$ nucleotide sequence identity. The novel FAdV-4 isolates were more distantly related to FAdV-A, FAdV-B, FAdV-D, and FAdV-E strains, with nucleotide identities determined at 76.0-76.3, 73.2-73.3, 69.6-71.7, and 74.8-75.1, respectively. 


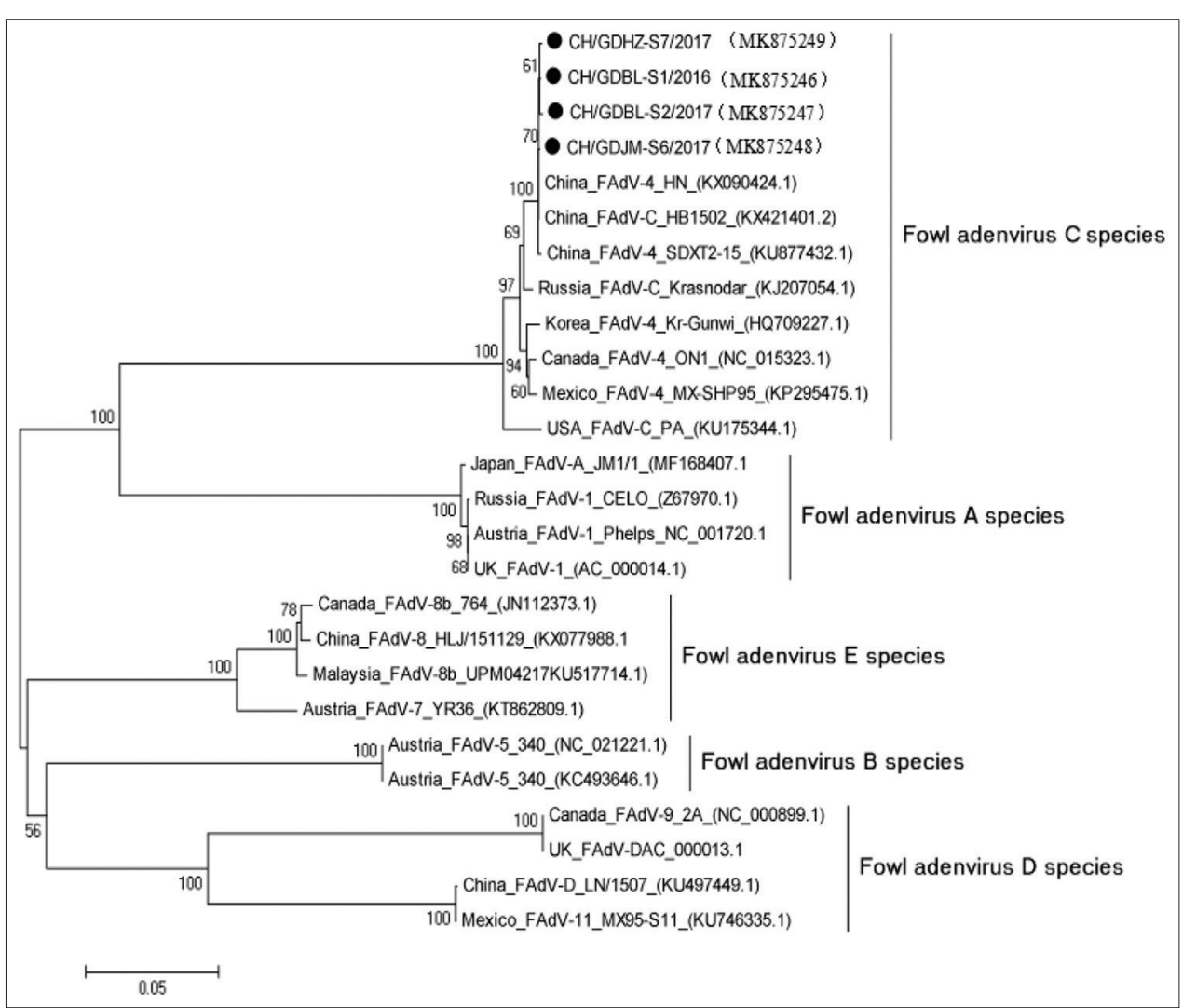

Figure-4: Phylogenetic analysis of nucleotide sequences of the hexon genes.

A phylogenetic tree, based on the sequences of the hexon genes, generated five distinct clusters. As anticipated, the novel FAdV-4 strains clustered with those identified as FAdV-C; the hexon genes from FAdV-A, FAdV-B, FAdV-D, and FAdV-E strains generate distinct clusters, similar to results determined in the previous studies [17].

The hexon protein is the main surface antigen of avian adenovirus; its epitopes are comparatively conserved, and there is a high degree of sequence conservation among strains. We identified only nine AA divergence sites in our comparison of the new isolates to the GenBank reference sequence. Further research will be needed to determine the relationship between nucleotide sequence variation, virulence, and outbreak frequency. These results highlight the conservation of the hexon protein as a target antigen for vaccine development.

\section{Conclusion}

Infections with FAdV-4 have had an enormous impact on the poultry industry. Here, we explored the molecular epidemiology and characteristics of an outbreak of HPS at poultry farms in Guangdong, China. The viruses, isolated from infected chickens, were identified as FAdV serotype-4, of the subgroup FAdV-C. Sequence analysis revealed a high degree of conservation among the new isolates and the reference strains for this serotype. Since our results were based on a limited number of samples, evaluation of a more extensive set of virus isolates will be needed to confirm these preliminary findings.

\section{Authors' Contributions}

Conceptualization, HS, SE, and LZ; formal analysis, FY, YS, DW, CS, LW, HW, LX, KM, GJ, ZX, and LZ; investigation, FY, YS, DW, CS, LW, HW, LX, KM, GJ, ZX, and LZ; methodology, FY and YS; supervision, HS; and writing-review and editing, HS, SE, and LZ; . All authors have read and agreed to the published version of the manuscript.

\section{Acknowledgments}

This work was supported by the National Natural Science Foundation of China (No. 31502071), Youth Innovative Talents Project of Guangdong Province Education Department, China (No. 2017KQNCX212), Guangdong province, China (2017GDK07), Start-up Research Grant Program provided by Foshan University, Foshan city, Guangdong Province for distinguished researchers, Guangdong Science and Technology Plan Project, China (Grant No: $1244060045607389 X C$ ), and School of Life Science and Engineering fund, China (Grant No: KLPREAD201801-02).

\section{Competing Interests}

The authors declare that they have no competing interests.

\section{Publisher's Note}

Veterinary World remains neutral with regard to jurisdictional claims in published institutional affiliation. 


\section{References}

1. Niu, Y.J., Sun, W., Zhang, G.H., Qu, Y.J., Wang, P.F., Sun, H.L., Xiao, Y.H. and Liu, S.D. (2016) Hydropericardium syndrome outbreak caused by fowl adenovirus serotype 4 in China in 2015. J. Gen. Virol, 97(10): 2684-2690.

2. Fauquet, C.M., Mayo, M.A., Maniloff, J., Desselberger, U. and Ball, L.A. (2004) Virus Taxonomy: Eighth Report of the International Committee on Taxonomy of Viruses. Viruses, 83(12): 988-992.

3. Li, X.J., Wu Che, Lei Xiaoya, Yan Yiming, Dai Zhenkai and Zhang Xinheng (2017) Heart-shaped package effusion isolation and identification and phylogenetic analysis of the pathogen of comprehensive syndrome. Prog. Vet. Med., 38(2): 27-31.

4. Yuan, W.Z., Li, Y.B., Wang, J.C., Zhang, S., Chen, P., Cui, Y., Jing, M., Li, J.N., Zuo, Y., Dong, S.S., Chen, L.G., Liu, J.X. and Sun, J.G. (2016) Heart-shaped package effusion a preliminary study on hepatitis syndrome. Chin. Vet. Sci., 46(2): 157-160.

5. Dahiya, S., Srivastava, R.N., Hess, M. and Gulati, B.R. (2002) Fowl adenovirus serotype 4 associated with outbreaks of infectious hydropericardium in Haryana, India. Avian Dis., 46(1): 230-233.

6. Ye, J., Liang, G., Zhang, J., Wang, W., Song, N., Wang, P., Zheng, W., Xie, Q., Shao, H., Wan, Z., Wang, C., Chen, H., Gao, W. and Qin, A. (2016) Outbreaks of serotype 4 fowl adenovirus with novel genotype, China. Emerg. Microbes Infect., 5(1): 5-50.

7. Zhang, T., Jin, Q., Ding, P., Wang, Y., Chai, Y., Li, Y., Liu, X., Luo, J. and Zhang, G. (2016) Molecular epidemiology of hydropericardium syndrome outbreak associated serotype 4 fowl adenovirus isolates in central China. Virol. J., 13(1): 188.

8. Zhu, Y.Q., Zhang, J.P., Xie, H.Y., Yang Desheng, Chen Jianhong, Zheng Jianbo. (2009) Study on detection method of HI antibody against avian influenza in waterfowl. Chin. J. Vet. Med., 45(2): 42-43.
9. Zhang Muhui (2016) Comparison of detection methods of avian leukemia in ephedra chicken flocks and observation of histopathology [D]. South China Agricultural University, CLC number: S858.31

10. El-Ashram, S., Al Nasr, I. and Suo, X. (2016) Nucleic acid protocols: Extraction and optimization. Biotechnol. Rep., 5(12): 33-39.

11. Chang, W.S. and Wang, T. (2015) Heart-shaped package effusion syndrome (Ankara disease) pathogen detection. China Anim. Healthc., 17(12): 25-26.

12. Abe, T., Nakamura, K., Tojo, H., Mase, M., Shibahara, T., Yamaguchi, S. and Yuasa, N. (1998) Histology, immunohistochemistry, and ultrastructure of hydropericardium syndrome in adult broiler breeders and broiler chicks. Avian Dis., 42(3): 606-612.

13. Mendelson, C., Nothelfer, H.B. and Monreal, G. (1995) Identification and characterization of an avian adenovirus isolated from a spiking mortality syndrome field outbreak in broilers on the Delmarva Peninsula, USA. Avian Pathol., 24(4): 693-706.

14. Shah, M.S., Ashraf, A., Khan, M.I., Rahman, M., Habib, M., Chughtai, M.I. and Qureshi, J.A. (2017) Fowl adenovirus: History, emergence, biology and development of a vaccine against hydropericardium syndrome. Arch. Virol., 162(7): 1833-1843.

15. Liu, D.Z. (2015) Prevention and treatment of hydronephrosis hepatitis syndrome. China Anim. Healthc., 17(10): 49-50.

16. Changjing, L., Haiying, L., Dongdong, W., Jingjing, W., Youming, W., Shouchun, W., Jida, L., Ping, L., Jianlin, W., Shouzhen, X., Shangjin, C., Yi, Z. and Yanbo, Y. (2016) Characterization of fowl adenoviruses isolated between 2007 and 2014 in China. Vet. Microbiol., 197(25): 62-67.

17. Li, L., Luo, L., Luo, Q., Zhang, T., Zhao, K., Wang, H., Zhang, R., Lu, Q., Pan, Z., Shao, H., Zhang, W. and Wen, G. (2016) Genome sequence of a fowl adenovirus serotype 4 strain lethal to chickens, isolated from China. Genome Announc., 4(2): e00140-16. 